\title{
Antibiotics Overuse and Bacterial Resistance
}

\author{
Mir Saleem*, Brett Deters, Adam de la Bastide and Martha Korzen \\ Department of Biology, Halmos College of Natural Sciences and Oceanography, NOVA Southeastern University, USA
}

\begin{abstract}
Antibiotic usage has become very widespread, as they are used to treat so many infectious diseases today. Antimicrobial agents exert their actions via different mechanisms including blockage of cell wall synthesis, interference of protein and/ or nucleic acid synthesis, interruption of cell membrane structure, and inhibition of a metabolic pathway. The treatment of bacterial infections with antimicrobial agents has become more difficult due to the capability of bacteria to develop resistance to antibiotics. Erroneous diagnosing, misconceptions, and improper physician-patient dynamics have led to overuse of antibiotics and the emergence of drug-resistant bacteria. Bacterial colonies have been shown to confer advantageous genetic information with ease, which is cause for concern. This, in turn, leads to a heightened urgency to create new forms of treatment that are effective against a greater proportion of a given bacterial colony. Effective ways of decreasing resistance include better diagnostic techniques, proper education and assessments, optimization of antibiotics usage, drug synergism, vaccine implementation, global efforts to combat resistance, and development of new antimicrobial agents.
\end{abstract}

\section{Keywords}

Antibiotics, Overuse, Viral infection, Bacterial infection, Broad-spectrum agents, Resistance

\section{Introduction}

Pathogenic microorganisms have negatively affected human health throughout history. A significant percentage of human population has been affected by bacterial infections (e.g., bubonic plague and tuberculosis) and non-bacterial infections (e.g., malaria and HIV) pandemics [1].

With the discovery of penicillin and some other classes of antibiotics in twentieth century, they were considered miracle drugs. However, in the twenty-first century, antibiotics are still being used, but with much less success. Many factors contributed to antibiotic resistance that made the antibiotics less effective.

\section{Contributing Factors for Bacterial Antibiotic Resistance}

\section{Antibiotics overuse}

Antibiotics are now being prescribed more frequently than ever before. The overuse of antibiotics evidently influences the evolution of resistance [2]. A direct relationship between antibiotic use as well as the rise and spreading of resistant bacterial strains exists $[2,3]$.

\section{Lack of regulations}

In many countries, antibiotics are unregulated and available over the counter without a prescription. This lack of regulation results in antibiotics that are easily accessible, plentiful, and cheap, which promotes overuse. Even in countries where antibiotics are regulated, they are available for online purchase $[3,4]$.

\section{Lack of optimization}

In $30-50 \%$ of patients, treatment indication, choice of antibiotic, or duration of therapy is inappropriate $[5,6]$. Incorrectly prescribed antibiotics have questionable therapeutic benefit and expose patients to potential complications of antibiotic therapy [7].

In an outpatient setting, antibiotics are most often prescribed for respiratory tract infections, which are caused by viruses. However, antibiotics usage continues to increase despite it provides little benefit [8]. Antibiotics overuse for respiratory tract infections were more common in the private sector than in the public sector [9].

*Corresponding author: Mir Saleem, MD, MS, Department of Biology, Halmos College of Natural Sciences and Oceanography, NOVA Southeastern University, $3^{\text {rd }}$ Floor, Parker Building, 3301 College Avenue, Fort Lauderdale, FL 33314, USA, Tel: 954-2628360

Accepted: October 24, 2019

Published online: October 26, 2019

Citation: Saleem M, Deters B, Bastide ADL, et al. (2019) Antibiotics Overuse and Bacterial Resistance. Ann Microbiol Res 3(1):93-99 


\section{Factors associated with healthcare professionals}

Physicians tend to use broad-spectrum agents with the wrong dose and frequency for the majority of common infections [10]. Incorrect prescribing of antibiotics can be caused by errors in diagnosis. A research study of patients with urinary tract infections showed that the physicians do not prescribe antibiotics based on specific signs and symptoms of UTI because of lack of proper documentation, and inadequate knowledge of proper antimicrobial agents [11]. In some countries like India, pharmacists copy doctor's prescriptions, then prescribe and dispense antibiotics themselves for respiratory tract infections [9]. Specialist physicians prescribe antibiotics less often because they have updated knowledge about the indication of antibiotics and access to government facilities with large infrastructures. Antibiotics are most commonly prescribed in rural areas. The practice of overprescribing is less common in the private urban setting and least common in a public setting [12].

\section{Factors associated with patients}

The biggest section of population for heavy antibiotic users are children and elderly [10]. A patient's knowledge and assumption about a specific illness may influence the expectations to receive antibiotics. The expectation increases if the patient assumes to have a bacterial infection [13]. Younger patients with a higher socioeconomic status are associated with antibiotic overuse [13]. The majority of patients believe that their risk of bacterial resistance increases if they take an antibiotic on a regular basis and they are not at risk if they are not on any antibiotics [14]. A survey of knowledge of antibiotics and antibiotic resistance in France showed that despite media awareness campaigns, the overall public knowledge remains low [15]. Some studies show that patients are more satisfied with a careful clinical examination, explanation of diagnosis, reassurance by the physician, and a discussion about the reason for antibiotic prescription $[9,13]$.

\section{Doctor-patient relationship}

Surveys show that physicians overprescribing antibiotics for many reasons including patients' satisfaction, fear of patients' health deterioration, keeping the patients from leaving for another doctor, patients' expectation to receive antibiotics, uncertainty in diagnosing the infection as viral or bacterial, lack of established doctor-patient relationships, pressure from patients, work responsibilities, and the personalities of physicians. Older and the more senior physicians that were working in private practice were more likely overprescribing antibiotics. In addition, doctors in private practice are prescribing more often to keep patients, while the doctors in public practice are overprescribing in order to save more time due to their busy schedule $[8,9,16]$.

\section{Use in agriculture}

In many countries around the world, antibiotics are used in livestock to promote growth and prevent infection. The agricultural use of antibiotics also affects the environmental microbiome. Most of the antibiotics given to livestock are excreted in urine and stool, which is dispersed through fertilizer, groundwater, and surface runoff [3,5,17-19].

\section{Use of cleaning products}

Some hygienic and cleaning products contain antibacterial products, which adversely affect the development of immunities to environmental antigens in both children and adults. This will have negative effects on immune system and the normally non-virulent microorganisms may increase morbidity and mortality due to infections $[4,20]$.

\section{Setting}

MRSA is becoming more common in intensive care units, and its importance has more than doubled in ten years [21]. While nosocomial strains have slowly been decreasing, community-acquired strains have been gradually expanding due to increase in mobility. With frequent domestic and international travel, the spread of diseases, including MRSA, has enhanced rapidly [21]. Drug-resistant E. coli infections have become more prevalent in hospitals, and there is a rise in drug-resistant infections in members of the public that had limited contact with the hospital setting. Studies shows that antibiotic-resistant E. coli infections have doubled, and people with little healthcare contact contracting the disease faster than those that have regular contact. Between 2009 and 2014, there has been more than a threefold increase in community-acquired infections [14]. C. difficile is the inflammation of the colon due to a bacterial infection and is linked with prior antibiotic use. In recent years, an antibiotic-resistant strain has been spreading; causing more serious disease, failure in treatment, and death [22].

\section{Mechanisms of Antibiotics Resistance}

In bacteria, genes can be inherited from relatives or attained from nonrelatives. This horizontal gene transfer (HGT) can allow antibiotic resistance to be transferred among different species of bacteria. Resistance can also occur spontaneously through mutation $[1,2]$.

\section{Antibiotics targeting cell wall}

Antibacterial agents can inhibit cell wall synthesis by interfering with the enzymes required for the synthesis of the peptidoglycan layer or by binding to the terminal D-alanine residues of the nascent peptidoglycan chain. These antibiotics include $\beta$-lactams and the glycopeptides [23-25]. Bacteria have developed different resistance mechanisms against this class of antibiotics. $\beta$-lactamases hydrolyze nearly all $\beta$-lactams that have ester and amide bond [26].

Class A $\beta$-lactamases are penicillinase and are clavulanic acid susceptible, which are found in members of Enterobacteriaceae [27].

Class B $\beta$-lactamases are resistant to inactivation by clavulanate, sulbactam, aztreonam, and carbapenems [28].

With exception of Salmonella and Klebsiella, all Gram-negative bacteria produce class $C \beta$-lactamases, which are immune to all $\beta$-lactams except carbapenems. They are not in- 
hibited by clavulanate $[29,30]$.

Class D $\beta$-lactamases are found in Enterobacteriaceae and in $P$. aeruginosa. These bacteria produce oxacillin-hydrolyzing enzymes, which give resistance to penicillin, cloxacillin, oxacillin, and methicillin. They are weakly inhibited by clavulanic acid [31].

\section{Inhibitors of protein biosynthesis}

Antimicrobial agents inhibit protein biosynthesis by targeting the $30 \mathrm{~S}$ or $50 \mathrm{~S}$ subunit of the bacterial ribosome. Aminoglycosides and tetracyclines inhibit $30 \mathrm{~S}$ ribosomal subunit. Chloramphenicol, macrolides, and oxazolidinones inhibit $50 \mathrm{~S}$ subunit of ribosome. Alteration of $30 \mathrm{~S}$ and $50 \mathrm{~S}$ ribosomal subunits affects protein synthesis and results in antibiotic resistance [32-36].

S. aureus, E. faecalis, and S. pneumoniae strains produce aminoglycoside-modifying enzymes (AMEs), which neutralize aminoglycosides by decreasing the affinity of a modified molecule and disrupting the binding to the 30 S ribosomal subunit $[37,38]$.

In tetracycline resistance, tet genes encode efflux proteins in Gram-positive and Gram-negative bacteria and affect new membrane transporters [39].

Chloramphenicol transacetylase enzyme is produced by some Gram-positive and Gram-negative bacteria and some of Hemophilus influenzae strains, which prevents the modified chloramphenicol from binding to a ribosomal 50 S subunit [40].

In macrolide resistance, erm-encoded methylases in S. aureus, S. pneumoniae, and $S$. pyogenes cause methylation of ribosomal active site with reduced binding. In addition, Mef type pump has been explained as a mechanism of resistance for S. pneumoniae, and S. pyogenes against macrolides [39].

In oxazolidinones resistance, E. faecium and $S$. aureus cause mutation of target site, which leads to reduced binding to active site [39].

\section{Folic acid metabolism inhibitors}

Sulfonamides and trimethoprim inhibit specific steps of folic acid metabolism in bacteria [41]. E. coli, S. aureus and S. pneumoniae cause mutation of genes encoding Dihydropteroate synthase (DHPS) [39].

\section{Interference with nucleic acid synthesis}

Fluoroquinolones inhibit DNA synthesis by creating double-strand DNA breaks during DNA replication, and Rifampin inhibits RNA synthesis [1,42].

Enteric Gram-negative bacteria and S. aureus cause mutations in gyr $A$ that results in decreased binding to active site(s). Mutations in gyr A and par C in S. pneumoniae, and Nor-A in S. aureus affect membrane transporters [1,39].

\section{Disruption of bacterial membrane structure}

Examples of this class include polymyxins and daptomycin [39]. Polymyxins increase bacterial membrane permeability, which results in the leakage of bacterial contents. Daptomycin inserts its lipid tail into the bacterial cell membrane, causing lethal bacterial membrane depolarization $[43,44]$.

Resistance to polymyxins is seen in some Gram-negative species, such as Neisseria meningitidis, Proteus mirabilis and Burkholderia spp. These bacteria change the lipopolysaccharide (LPS) structure, as polymyxins initially interact with the negatively charged lipid A component of LPS [45].

\section{Role of Antibiotics on Microenvironment and Immune Response}

Broad-spectrum antibiotics have a multitude of effects on the microenvironment of mammalian cells. Antibiotics can have negative impacts on mitochondrial function, which may translate to compromised metabolism and cellular respiration. This was seen in a 2017 paper which showed that the normal electron transport and respiratory activity in mouse macrophages are inhibited by common antibiotics. This finding also has further implications. Antibiotics may have tissue-specific effects on different individuals, depending on their dynamic and personal microenvironment. An example of this potential specificity was demonstrated when data indicated a depletion of metabolic intermediates in the peritoneum of mice. The study then went on to see how metabolic inhibition would affect immune cells like phagocytes. Overall, the phagocytic killing of pathogens and the metabolic potential of common immune cells that respond to sites of infection was diminished. By inhibiting normal metabolism in cells, antibiotics can impede the production of necessary secondary messengers that play vital roles in immune and inflammatory cascades. The metabolic changes made in the host by antibiotics can ultimately lead to a decrease in the drug's efficacy. This study also reiterated the negative changes in the microbiomes of hosts taking antibiotics [46].

Patients that are frequently prescribed broad-spectrum antibiotics may be at risk of suppressing their immune system. It is well understood that the brunt of the immune system is located in the gut and is formed by the microbiome. Ironically, prescription antibiotics that are designed to treat illness and infection may compromise the immune response in some patients, if they are used gratuitously. This is discussed in a 2013 paper by Ubeda and Pamer, which contends that antibiotics will impact the production of antibodies, T-cell differentiation, and the expression of antimicrobial peptides that are found in intestinal epithelial cells. These effects, in concert, can leave the immune system vulnerable to subsequent attacks [47].

Another study focused on the effects of antibiotics on white blood cell count and sought to determine if the change in WBC count was due to the normal rebound of the immune system, or if it was directly caused by common antibiotics. Total WBC count of participants was compared before and after antibiotics were administered. According to their results, certain antibiotics can diminish absolute neutrophil count, which is the most prevalent of the five classes of WBCs [48]. 


\section{Cost of Antibiotic Resistance}

Health care cost significantly increase due to antibiotic-resistant infections. Physicians have to use the more toxic and more expensive antibiotics to fight against bacterial infections $[5,7]$. Patients with resistant infections stay in the hospital 6.4 to 12.7 days longer, require more doctors' visits, and suffer a higher rate of long-term disability $[5,20]$. Antibiotic-resistant infections economic burden is as high as \$20 billion in health care costs and $\$ 35$ billion a year in lost productivity. In addition, the burden of families and communities increases due to lost wages and health care costs $[4,20]$.

\section{The Solution}

New treatments and therapies are constantly being developed in order to overcome drug-resistant infections.

\section{Vaccination}

Vaccination is beneficial in preventing or reducing the incident of certain bacterial infections. The vaccination programs against Haemophilus influenzae and Streptococcus pneumoniae have been very effective in significantly reducing bacterial infection; however, there are some concerns about the emergence of bacterial resistance against these vaccines. Use of pneumococcal vaccines in children and elderly have dramatically reduced the occurrence of otitis media, sinusitis, pneumonia, sepsis, and meningitis. Through the use of these vaccinations, the rate of prescribing antibiotics has been declined $[49,50]$. Despite of widespread efforts to prevent MRSA, it has been tremendously difficult to develop a staphylococcal vaccine. Development of vaccines against $\beta$-lactamases and transpeptidases in $\beta$-lactam-resistant bacteria has been difficult so far [51].

\section{Patients education}

Patient counselling usually have a positive impact on reducing antibiotic overuse. Physician should emphasize patient compliance with antibiotic treatment. To increase compliance, doctors should pay attention to the cost of medications, must maintain good communication with patients, and provide appropriate educational materials for them to read [52].

The groups of patients with higher levels of misconceptions and lower levels of knowledge about the negative impact of antibiotics usage include persons of lower socioeconomic status, lower educational status, males, those in younger age groups, and the elderly. These patients should be a part of any educational efforts to improve their knowledge about antibiotic misuse and reduce their expectations to receive antibiotic prescriptions when it is not clinically indicated [53].

\section{Role of healthcare professionals}

Antibiotic misuse can be controlled by providing the physician with proper indications and information, a critical analysis of data, a description of basic antibacterial activity, and information regarding the epidemiology of acquired resistance [54].

Doctors should be able to crossmatch culture samples and see what drug the infection is susceptible to. Pharmacists can see if a culture is resistance to a particular antibiotic and can recommend a different one [51]. In response to the increase of antibiotic-resistant bacteria, more hospitals have created programs to help physicians and pharmacists limit the use of antibiotics [51].

To reduce misconceptions and misguided expectations about the treatment with antimicrobial agents, it is important to improve the patient's knowledge and attitude about the proper use of antibiotics [52]. A study in China showed that when performance data of physicians are available for public to view, the physicians appropriate prescribing behavior gradually improves [55].

\section{Diagnostic techniques}

Before starting an empiric therapy with antibiotics, cultures should be obtained, and the cultures should be used to assist the physician in adjusting therapy. It is essential to obtain a clean culture from sterile sites before the start of antimicrobial agents. If the culture is negative from the sterile site and the patient remains sick, supplementary tests e.g., CBC and differential or C-reactive protein should be obtained to guide the treatment [56].

In order to correctly diagnose an infection, several tests must be conducted in conjunction with applied physician intuition, as no single test can diagnose an infection with total accuracy [57].

A genetic study concluded that no single gene can account for the presence of resistance in bacteria. Whole-genome sequencing can be used to understand the pathology of certain bacteria, in conjunction with diagnostic analysis, susceptibility, and current epidemiological research [58].

\section{Reduction in use}

Restriction of certain antibiotics in humans does decrease resistance, but it also increases the use of and the resistance to other antibiotics that are used instead. Continued rotation of antibiotics can help decrease resistance, but only within the facility [59].

Reduction in use of antibiotics in livestock results in a substantial decrease of resistant bacteria in livestock and humans; and the restriction does not have any negative effect on the production levels [60]. To reduce antibiotics overuse, fees can be implemented for agricultural use. The fees could be used for research and development of new methods of antibacterial treatment [61].

\section{Optimization of use}

The empiric therapy protocol should be standardized. If possible, use of broad-spectrum antibiotics should be minimized [56]. Antibiotics should be prescribed only to treat bacterial infections. Evaluation of biological markers may be necessary in some cases [61]. There is no need for prophylaxis against urinary tract infections, as they are treated easily and are generally rare [62]. Effective antibiotic prescribing requires proper drug selection, dosing, route of administration, and interval [63]. For many antibi- 
otics, careful attention to serum levels is also required to improve efficacy while reducing toxicity [56].

\section{Route of administration}

When IV antibiotics are used only for serious infections, overuse of antibiotics can be prevented in unnecessary cases. The enteral route is the preferred option for less serious infections. Switching to enteral after IV may also be done. Intubation increases risk of infection and should also be avoided when possible. Duration of parenteral nutrition should be decreased and must be replaced with enteral nutrition when it is clinically possible [59]. Skin infections should be treated until the condition is cured. Drainage and debridement are required for invasive tissue infections before providing antibiotics. Vascular catheters increase risk of infection and they should be intermittently removed or inserted in a different location [64].

\section{Monotherapy vs. combined therapy}

There are conflicting studies about the effectiveness of monotherapy vs. combined therapy. Some other studies show that combination therapy is no more effective in fighting superbugs than using a single antibiotic, while one experiment showed that monotherapy was linked with a smaller risk of infection. Another study shows that combination therapy has a reduced risk of resistance [65].

It is not always necessary to combine two gram-negative fighting antibiotics unless resistance levels happen to be high. Once the susceptibility of infection is known, the treatment should ideally be continued with only one antibiotic. The duration should be noted, for studies show that a shorter duration had a similar mortality rate, although there is a significant decrease in the rise of resistance [49].

Combined treatment with piperacillin, tazobactam, and meropenem, shows beneficial results in patients infected with MRSA. In addition, this combination didn't produce resistance against the drug [14]. In a study involving the efficacy of doxycycline and benzoyl peroxide on treatment of acne, researchers found that antibiotics in combination with benzoyl peroxide can increase the effectiveness of treatment by reducing the necessary dosage or duration of antibiotic therapy [66].

\section{Alternative options}

Ultraviolet germicidal irradiation (UVGI) is an effective disinfectant against MRSA and can help facilitate healing wounds. However, the research was only on two strains of MRSA, so more research is needed to see if all strains show the same results [67].

$\mathrm{NaOCl}$ or bleach is effective against viruses, bacteria, spores, and fungi. So far, no bacterial resistance has been found. It is also inexpensive and widely available, allowing it to be a better treatment option for certain infections. Bleach baths is beneficial against infected atopic eczema and can be used to replace antibiotics [68].

\section{Current global efforts}

CDC recommends that countries around the world should create and implement a comprehensive plan to deal with antimicrobial resistance in healthcare, food industry, communities, soil and water. These plans should include infection prevention and control practices, appropriate access and use of antibiotics, bacterial resistance tracking systems, and improvement in diagnostic techniques. Global effort should also concentrate on developing new drugs, diagnostic methods, vaccines, and therapeutics to treat infections [69].

In response to increasing drug-resistant bacteria strains, the World Health Organization called for a global response to the problem. The World Health Assembly recommended a global action plan to challenge antimicrobial resistance. This plan has five specific objectives, which include increase awareness and understanding of antimicrobial resistance, enhance knowledge using surveillance and research, decrease the incidence of infection, improve the use of antimicrobial agents, and increase investment in new medicines, diagnostic tools, vaccines and other interventions [70].

\section{Future research studies}

Through gene manipulation, non-pathogenic antibiotrophs can be engineered for pharmaceutical, agricultural, and clinical applications. Their chemoattractant patterns could be employed to recognize antibiotic outflow, while their genomic profiles could help enhance CRISPR interference and could assist the next generation of molecular cloning utilization [71].

Bacterial repellents including bio toxic and microtoxic inhibitors may be better than the current bactericidal approach. Such cytotoxin neutralizers can be very effective since they do not affect colony viability. With this approach, these agents do not activate resistance-favorable mutations, ultimately decreasing the spread of resistant genes [72-74].

\section{Conclusion}

Antibiotics have been overprescribed due to poor patient and physician education, internal and external pressures, and increasing demands. The overuse of antibiotics has led to the emergence of drug-resistant strains of bacteria. World health organization, Center for Disease Control and Infectious Disease Society of America recognized bacterial resistance as a pandemic issue with disastrous consequences if the condition is not controlled in the near future. Our dependence on antibiotics for livestock and treatment of infectious diseases in humans are high. Unfortunately, the bacteria are evolving and becoming resistant to the available antimicrobial agents. There is an urgent need for development of new antibacterial agents. Education of physicians about the appropriate indications, proper dosages, and route of administration are important factors to prevent overuse of antibiotics. In addition, physicians should educate their patients about the risk of unnecessary antibiotic treatment. Global efforts to prevent infections and to invest in the new method of treatments are crucial.

Although, there are some solutions currently available to 
deal with multi-resistant bacteria, complete eradication of these bacteria seems very difficult at this time. A promising approach is use of various antibiotics with different mechanism of actions. Other solutions to fight drug-resistant strains and resistance overall include new diagnosing procedures, alternative treatments, vaccinations, bacterial genomics, and the restricted use of antibiotics for agricultural use. With a widespread commitment to resolving this problem, the imminence of antibiotic resistance can be left in the past.

\section{References}

1. Fred C Tenover (2006) Mechanisms of antimicrobial resistance in bacteria. Am J Infect Control 119: S3-S10.

2. Read AF, Woods RJ (2014) Antibiotic resistance management. Evol Med Public Health 2014: 147.

3. (2013) The antibiotic alarm. Nature 495: 141.

4. Michael CA, Dominey-Howes D, Labbate M (2014) The antibiotic resistance crisis: Causes, consequences, and management. Front Public Health 2: 145.

5. http://www.cdc.gov/drugresistance/threat-report-2013

6. Luyt CE, Brechot N, Trouillet JL, et al. (2014) Antibiotic stewardship in the intensive care unit. Crit Care 18: 480.

7. Lushniak BD (2014) Antibiotic resistance: a public health crisis. Public Health Rep 129: 314-316.

8. Arason VA, Sigurdsson JA (2010) The Problems of antibiotic overuse. Scand J Prim Health Care 28: 65-66.

9. Kotwani A, Holloway K (2014) Antibiotic prescribing practice for acute, uncomplicated respiratory tract infections in primary care settings in New Delhi, India. Trop Med Int Health 19: 761-768.

10. Malo S, Rabanaque MJ, Feja C, et al. (2014) High antibiotic consumption: A characterization of heavy users in Spain. Basic Clin Pharmacol Toxicol 115: 231-236.

11. Kistler CE, Zimmerman S, Scales K, et al. (2017) The antibiotic prescribing pathway for presumed urinary tract infections in nursing home residents. J Am Geriatr Soc 65: 1719-1725.

12. Kumar R, Indira K, Rizvi A, et al. (2008) Antibiotic prescribing practices in primary and secondary health care facilities in Uttar Pradesh, India. J Clin Pharm Ther 33: 625-634.

13. Haltiwanger KA, Hayden GF, Weber T, et al. (2001) Antibioticseeking behavior in college students: What do they really expect. J Am Coll Health 50: 9-13.

14. Lenhoff A (2016) In the news: Antibotic resistance. MLO: Medical Laboratory Observer 26-27.

15. Robert A, Nguyen Y, Bajolet O, et al. (2017) Knowledge of antibiotics and antibiotic resistance in patients followed by family physicians. Med Mal Infect 47: 142-151.

16. Lam TP, Lam KF (2003) What are the non-biomedical reasons which make family doctors over-prescribe antibiotics for upper respiratory tract infection in a mixed private/public asian setting. J Clin Pharm Ther 28: 197-201.

17. Spellberg B, Gilbert DN (2014) The future of antibiotics and resistance: a tribute to a career of leadership by John Bartlett. Clin Infect Dis 59: S71-S75.

18. Gross M (2033) Antibiotics in crisis. Current Biology 23: 10631065.
19. Bartlett JG, Gilbert DN, Spellberg B (2013) Seven ways to preserve the miracle of antibiotics Clin Infect Dis 56: 1445-1450.

20. Golkar Z, Bagazra O, Pace DG (2014) Bacteriophage therapy: A potential solution for the antibiotic resistance crisis. J Infect Dev Ctries 8: 129-136.

21. Dragon N (2006) Fighting today's superbugs. Australian Nursing Journal 14: 16-19.

22. (2008) C. difficile rates in hospital patients higher than expected. Hospitals \& Health Networks 1: 47.

23. Džidic S, Šuškovic J, Kos B (2008) Antibiotic resistance mechanisms in bacteria: Biochemical and genetic aspects. Food Technol Biotechnol 46: 11-21.

24. Grundmann H, Aires de Sousa M, Boyce J, et al. (2006) Emergence and resurgence of meticillin resistant Staphylococcus aureus as a public health threat. Lancet 368: 874-785.

25. McManus MC (1997) Mechanisms of bacterial resistance to antimicrobial agents. Am J Health Syst Pharm 54: 1420-1433.

26. Alekshun MN, Levy SB (2007) Molecular mechanisms of antibacterial multidrug resistance. Cell 128: 1037-1050.

27. Rice LB, Sahm D, Bonomo R (2003) Mechanisms of resistance to antibacterial agents. In: Murray PR, Manual of Clinical Microbiology. ( $8^{\text {th }}$ edn), ASM Press, Washington, DC, 1084-1087.

28. Rasmussen BA, Bush K (1997) Carbapenem hydrolyzing beta lactamases. Antimicrob Agents Chemother 41: 223-232.

29. Crichlow GV, Kuzin AP, Nukaga M, et al. (1999) Structure of the extended spectrum class $\mathrm{C}$ beta lactamase of Enterobacter cloacae GC1, a natural mutant with a tandem tripeptide insertion. Biochemistry 38: 10256-10261.

30. Lobkovsky E, Billings EM, Moews PC, et al. (1994) Crystallographic structure of a phosphonate derivative of the Enterobacter cloacae P99 cephalosporinase: Mechanistic interpretation of a beta lactamase transition state analog. Biochemistry 33: 67621672.

31. Naas T, Nordmann P (1999) OXA type beta lactamases. Curr Pharm Des 5: 865-879.

32. Vannuffel P, Cocito C (1996) Mechanism of action of streptogramins and macrolides. Drugs 1: 20-30.

33. Johnston NJ, Mukhtar TA, Wright GD (2002) Streptogramin antibiotics: Mode of action and resistance. Curr Drug Targets 3: 335-344.

34. Wise $R$ (1999) A review of the mechanisms of action and resistance of antimicrobial agents. Can Respir J 20-22.

35. Lambert PA (2005) Bacterial resistance to antibiotics: Modified target sites. Adv Drug Deliv Rev 57: 1471-1485.

36. Bozdogan B, Appelbaum PC (2004) Oxazolidinones: Activity, mode of action, and mechanism of resistance. Int J Antimicrob Agents 23: 113-119.

37. Strateva T, Yordanov D (2009) Pseudomonas aeruginosa A phenomenon of bacterial resistance. Journal of Medical Microbiology 58: 1133-1148.

38. Maurice F, Broutin I, Podglajen I, et al. (2008) Enzyme structural plasticity and the emergence of broad-spectrum antibiotic resistance. EMBO Rep 9: 344-349.

39. Kapoor G, Saigal S, Elongavan A (2017) Action and resistance mechanisms of antibiotics: A guide for clinicians. J Anaesthesiol Clin Pharmacol 33: 300-305. 
40. Tolmasky ME (2000) Bacterial resistance to aminoglycosides and beta lactams: The Tn1331 transposon paradigm. Front Biosci 5: 20-29.

41. Yoneyama H, Katsumata $R$ (2006) Antibiotic resistance in bacteria and its future for novel antibiotic development. Biosci Biotechnol Biochem 70: 1060-1075.

42. Drlica K, Zhao X (1977) DNA gyrase, topoisomerase IV, and the 4-quinolones. Microbiol Mol Biol Rev 61: 377-392.

43. Storm DR, Rosenthal KS, Swanson PE (1977) Polymyxin and related peptide antibiotics. Annu Rev Biochem 46: 723-763.

44. Carpenter CF, Chambers HF (2004) Daptomycin: Another novel agent for treating infections due to drug-resistant gram-positive pathogens. Clin Infect Dis 38: 994-1000.

45. Moffatt JH, Harper M, Boyce JD (2019) Mechanisms of polymyxin resistance. Adv Exp Med Biol 1145: 55-71.

46. https://doi.org/10.1016/j.chom.2017.10.020

47. Ubeda C, Pamer EG (2012) Antibiotics, microbiota, and immune defense. Trends Immunol 33: 459-466.

48. Shuman M, Lee Demler T, Trigoboff E, et al. (2012) Hematologic impact of antibiotic administration on patients taking clozapine. Innov Clin Neurosci 9: 18-30.

49. Hersh AL, Fleming-Dutra KE (2017) Vaccines and Outpatient Antibiotic Stewardship. Pediatrics 140.

50. Birgitta Henriques-Normark, Staffan Normark (2014) Bacterial vaccines and antibiotic resistance. Ups J Med Sci 119: 205-208.

51. McKinney M (2011) New Tools in Germ Warfare: Hospitals Turn to IT to Help Curb Overuse of Antibiotics, Fight Drug Resistance. Mod Healthc 41: 32-33.

52. Werk LN, Bauchner H (1998) Practical considerations when treating children with antimicrobials in the outpatient setting. Drugs 55: 779-790.

53. Vanden Eng J, Marcus R, Hadler JL, et al. (2003) Consumer Attitudes and Use of Antibiotics. Emerg Infect Dis 9: 1128-1135.

54. Schlemmer B (2001) Impact of registration procedures on antibiotic policies. Clinical Microbiology and Infection 7: 5-8.

55. Liu C, Zhang X, Wan J (2015) Public reporting influences antibiotic and injection prescription in primary care: A segmented regression analysis. J Eval Clin Pract 21: 597-603.

56. Cantey JB (2016) Optimizing the use of antibacterial agents in the neonatal period. Pediatr Drugs 18: 109-122.

57. Van De Geijn GJM, Denker S, Meuleman-Van Waning V, et al. (2016) Evaluation of new laboratory tests to discriminate bacterial from nonbacterial chronic pulmonary disease exacerbations. Int J Lab Hematol 38: 616-628.

58. Jindal HM, Ramanathan B, Le CF, et al. (2018) Comparative genomic analysis of ten clinical streptococcus pneumoniae collected from a malaysian hospital reveal 31 new unique drug- resistant snps using whole genome sequencing. J Biomed Sci 25: 1-14.

59. Richards GA (2005) The therapeutic challenge of gram-negative sepsis: Prolonging the lifespan of a scarce resource. Clin Microbiol Infect 11: 18-22.

60. Martin MJ, Thottathil SE, Newman TB (2015) Antibiotics overuse in animal agriculture: A call to action for health care providers. Am J Public Health 105: 2409-2410.

61. Cully M (2014) The politics of antibiotics. Nature 509: S16-S17.

62. Herr HW (2012) Outpatient Urological Procedures in AntibioticNative Patient with Bladder Cancer with Asymptomatic Bacteriuria. BJU Int 110: E658-E660.

63. Wade KC, Benjamin DK (2016) Clinical pharmacology of antiinfective drugs. In: Wilson $C B$, Nizet $V$, Maldonado $Y A$, Remington JS, Klein JO, editors. Remington and Klein's infectious diseases of the fetus and newborn infant. ( $8^{\text {th }}$ edn), Philadelphia, Elsevier.

64. Houghton D (2002) Blocking the path of antibiotic resistance. Nursing Management 33: 32.

65. Johnson SJ, Ernst EJ, Moores KG (2011) Is double coverage of gram-negative organism necessary. Am J Health-Syst Pharm 68: 119-124.

66. Chon SY, Doan HQ, Mays RM, et al. (2012) Antibiotic overuse and resistance in dermatology. Dermatol Ther 25: 55-69.

67. Green CF, Elbe LA, Neal TD, et al. (2015) Ultraviolet germicidal irradiation susceptibility of methicillin-resistant staphylococcus aureus compared with methicillin-susceptible S. aureus. Can J Microbiol 61: 871-875.

68. Barnes TM, Greive KA (2013) Use of bleach baths for the treatment of infected atopic eczema. Australas J Dermatol 54: 251-258.

69. https://www.cdc.gov/drugresistance/intl-actvities. html\#anchor_1534781904407

70. https://www.who.int/antimicrobial-resistance/global-actionplan/en/

71. Woappi Y, Gabani P, Singh A, et al. (2016) Antibiotrophs: The complexity of antibiotic-subsisting and antibiotic-resistant microorganisms. Crit Rev Microbiol 42: 17-30.

72. Baquero F, Tedim AP, Coque TM (2013) Antibiotic resistance shaping multi-level population biology of bacteria. Front Microbiol 4: 15.

73. Williams JJ, Halvorsen EM, Dwyer EM, et al. (2011) Toxinantitoxin (TA) systems are prevalent and transcribed in clinical isolates of pseudomonas aeruginosaand methicillin-resistant Staphylococcus aureus. FEMS Microbiol Lett 322: 41-50.

74. Williams JJ, Hergenrother PJ (2008) Exposing plasmids as the Achilles'heel of drug-resistant bacteria. Curr Opin Chem Biol 12: 389-399.

DOI: $10.36959 / 958 / 574$

Copyright: (C) 2019 Saleem M, et al. This is an open-access article distributed under the terms of the Creative Commons Attribution License, which permits unrestricted use, distribution, and reproduction in any medium, provided the original author and source are credited. 